\section{Residents need focused teach- ing during pediatric emergency medicine rotation to optimize their educational objectives}

\author{
Mohammed Alomar, ${ }^{1}$ Narges Daliri, ${ }^{1}$ \\ Awatif Alamer, ${ }^{1}$ Abdolmoneim Eldali ${ }^{2}$ \\ 'Emergency Department, King Faisal \\ Specialist Hospital and Research Centre, \\ Riyadh; ${ }^{2}$ Biostatistics Department, King \\ Faisal Specialist Hospital and Research \\ Centre, Riyadh, Saudi Arabia
}

\section{Abstract}

Pediatric Emergency Medicine (PEM) rotation provides a unique training environment for rotating residents. We aim to assess the impact of PEM rotation on the scientific knowledge of residents from different specialties and training centers by comparing the pre- and post-rotation knowledge. PEM Departments of three major tertiary care training centers were selected. Rotating pediatric and emergency medicine residents were given pre-test with twenty multiple-choice type questions related to the scientific knowledge of PEM and then re-tested with the same questions towards the end of their rotation. The $t$-test was used to compare mean scores. Further comparison based on specialty and training center was also done. Seventy-three residents were approached and enrolled, 48 from Pediatrics and 25 from Emergency Medicine. The mean pre- and post-scores for all residents were $15.9 / 20$ and 15.5/20, respectively. All residents' score was less on the post-rotation compared to the pre-rotation in all centers. Pediatric residents at one center scored higher, but they were not statistically significant. There were no statistically significant differences in resident specialty. We found a statistical difference between the residents of two centers compared to the third with $\mathrm{P}=0.04$ and 0.02 respectively. After one month of rotation in PEM, we observed a decrease in the post-rotation test scores as compared to the pre-rotation scores. Since the reasons for the lower scores could not be identified by this study, educational deficiencies should be identified and perhaps a focused teaching and allotted study time to optimize the residents educational objective could be advised.

\section{Introduction}

The Pediatric Emergency Medicine (PEM) rotation provides a unique training to a signif- icant number of rotating residents by offering them access to patients with undifferentiated medical issues. They are afforded continuous supervision by attending physicians. ${ }^{1-4}$ Over the past two decades, the knowledge about PEM subspecialty has grown through fellowship programs, formation of PEM sections in professional organizations and research. ${ }^{5-10}$ Pediatric and Emergency Medicine residents rotating in PEM are exposed to specific curriculum which has been developed by the Residency Training Committees. ${ }^{11-14}$

Saudi Commission for Health Specialties (SCFHS) in Saudi Arabia oversees all the training programs including Pediatrics, Emergency Medicine (EM) and has recognized and endorsed PEM subspecialty fellowship training program since January, 2005. ${ }^{15}$ Currently as per SCFHS Guidelines, Pediatric residents spend five months [ 2 months in first post-graduate year (PGY), 1 month on third and 2 months on the fourth PGY] in PEM; while EM residents have to spend 4 months (2 months on the second and 2 months on the fourth PGY). ${ }^{16}$ All residents are expected to participate and attend the didactic and practical training sessions and do at least eighteen clinical shifts per month during their rotation in PEM. We aim to assess the impact of PEM rotation on the scientific knowledge basis of residents from different specialties and centers by comparing their pre- and post-rotation knowledge.

\section{Materials and Methods}

A prospective, observational, and educational study of the impact of one month training in PEM at three different major tertiary care hospitals in Riyadh, Saudi Arabia on the residents' scientific knowledge was assessed by their performance on pre- and post-rotation written examinations.

\section{Study setting and population}

The study was conducted in the PEM Departments of three major tertiary care centers: King Faisal Specialist Hospital and Research Center (Hospital A), King Abdulaziz Medical City (Hospital B), and King Fahad Medical City (Hospital C). These hospitals were chosen due to their reputable established pediatric residency and PEM fellowship training programs and the presence of a relatively high number of trainees. Program directors were notified by the authors. Residents at PGY 1 to 4 in the training programs for Pediatric and Emergency Medicine that rotated in PEM as an elective or part of their integral program were selected.
Correspondence: Mohammed Alomar, Emergency Department, King Faisal Specialist Hospital and Research Centre, P.0. Box 3354 MBC 84, Takhassusi Street, 11211 Riyadh, Saudi Arabia. Tel. +966.1.4424425 - Fax: +966.1.4423429.

E-mail: momar@kfshrc.edu.sa

Key words: residents, education, pediatric, emergency.

Conflict of interests: the authors declare no potential conflict of interests.

Contributions: MA and ND, proposal writing; $\mathrm{AA}$, data collections; $\mathrm{AE}$, data analysis; MA and ND, manuscript writing.

Received for publication: 17 July 2013. Revision received: 11 September 2013

Accepted for publication: 25 September 2013.

This work is licensed under a Creative Commons Attribution 3.0 License (by-nc 3.0).

(C) Copyright M. Alomar et al., 2014

Licensee PAGEPress, Italy

Healthcare in Low-resource Settings 2014; 2:1831 doi:10.4081/hls.2014.1831

\section{Inclusion and exclusion criteria}

All rotating residents from Pediatric and Emergency Medicine training programs were included during the study period. There were no exclusions. We developed twenty multiplechoice type questions based on clinical case scenarios that cover resuscitation, emergent airway management, trauma care, toxicological and environmental emergencies with single best answer (Table 1). The questions were initially formulated by the principal investigator and subsequently approved by professionals in our institution interested in the field and the subject. The co-investigators and a few other PEM physicians were consulted to review, test the questions and suggest any modifications prior to final approval. The questions were distributed to all rotating residents in the three major hospitals at the commencement of their first shift and collected at the end of the same shift by the principal or co-investigators. All participants received the same 20 written questions in a paper format. After completion of the rotation participants again completed the same written examination. The residents were refrained from discussing the questions and urged to complete the test during the same shift. The participants were not given the correct answers or critical elements to the written test before, during, or after the rotation.

\section{Measurement and timetable}

The scores were given based on the correct responses out of 20 . The study was conducted 
over ten months from November 2011-July 2012.

\section{Hypothesis and outcomes}

The hypothesis is that residents shall show improvement in their score by the end of their rotation. Primary outcome is: change in the scientific knowledge of the residents by the end of their rotation. Secondary outcome is: any difference from one center or specialty compare to the other.

\section{Sample size and statistical analysis}

To our knowledge, based on literature search this is the first pilot study done in Saudi Arabia with participation of residents of different training programs rotating in PEM. We estimated that a meaningful difference in test performance would be at least $10 \%$ improvement in the score delta (post-test minus pretest). For detection of statistical significance, with two-tailed mean comparison test with $90 \%$ power and alpha of 0.05 , a sample size of at least 22 persons in each group was needed. This assumed a standard deviation (SD) of mean score delta to be $10 \%$. The student's $t$ test was used to compare mean test scores. Completed answers were entered in an Excel spreadsheet and SPSS version 10 statistical packages were used. Descriptive data used in form of frequency tables, which were generated for each resident in the test. Further comparison based on specialty and the training center was also done. A $\mathrm{P}<0.05$ was considered statistically significant.

\section{Ethics}

A verbal consent was taken from all participants and they were assured that the results of the tests would be kept confidential and have no bearing on their official evaluation or future performance in PEM. Office of Research Affairs' approval of Hospital A (base hospital of the investigators) approved the study before enrollment of subjects.

\section{Results}

All approached seventy-three residents from three centers were enrolled, 48 PEM and 25 EM. The exact number of residents enrolled from each center is indicated (Table 2). All residents completed the study during the enrollment period. The mean pre and post score for all residents were (15.9/20 and 15.5/20) respectively. None of the residents achieved full mark (20) in the pre- or post-rotation score. All residents scored less on post-rotation test compared to the pre-rotation except pediatric residents at Hospital B which was statistically not significant. There were no statistical differences in the means by residents' level within the centers. There were no statistically significant differences in resident specialty between the groups ( $>>0.05)$ (Table 3). As for the centers, there were a statistical difference among all residents at both Hospital $\mathrm{C}$ and Hospital A compared to Hospital B with a P value of 0.04 and 0.02 respectively (Table 4 ).

\section{Discussion}

Riyadh tertiary care hospitals are often thought to be ideal sites for PEM rotations because of high acuity and a diverse spectrum of patients. The clinical experience is some-

Table 1. Contents and distribution of the test questions.

\begin{tabular}{lc} 
Contents & $\begin{array}{c}\text { Number } \\
\text { of questions }\end{array}$ \\
\hline Dehydration & 2 \\
Metabolic disorders & 2 \\
\hline Upper airway emergencies & 2 \\
\hline Acute neurologic emergencies & 2 \\
\hline Simple laceration & 1 \\
Management of mammalian & 1 \\
bite wound & 1 \\
\hline Orthopedic emergencies & 1 \\
\hline Injury prevention advice & 1 \\
\hline Lower respiratory tract infection & 1 \\
\hline Immunocompromised with fever & 1 \\
\hline Management of soft tissue infection & 1 \\
\hline Sickle cell disease & 1 \\
\hline Acid-base imbalance & 1 \\
\hline Common congenital heart diseases & 1 \\
\hline Differential diagnosis of common & 1 \\
pediatric rashes & 1 \\
\hline Management of common & \\
toxic ingestion & \\
\hline
\end{tabular}

Table 2. Exact number of residents enrolled from each center with their pre- and post-rotation scores.

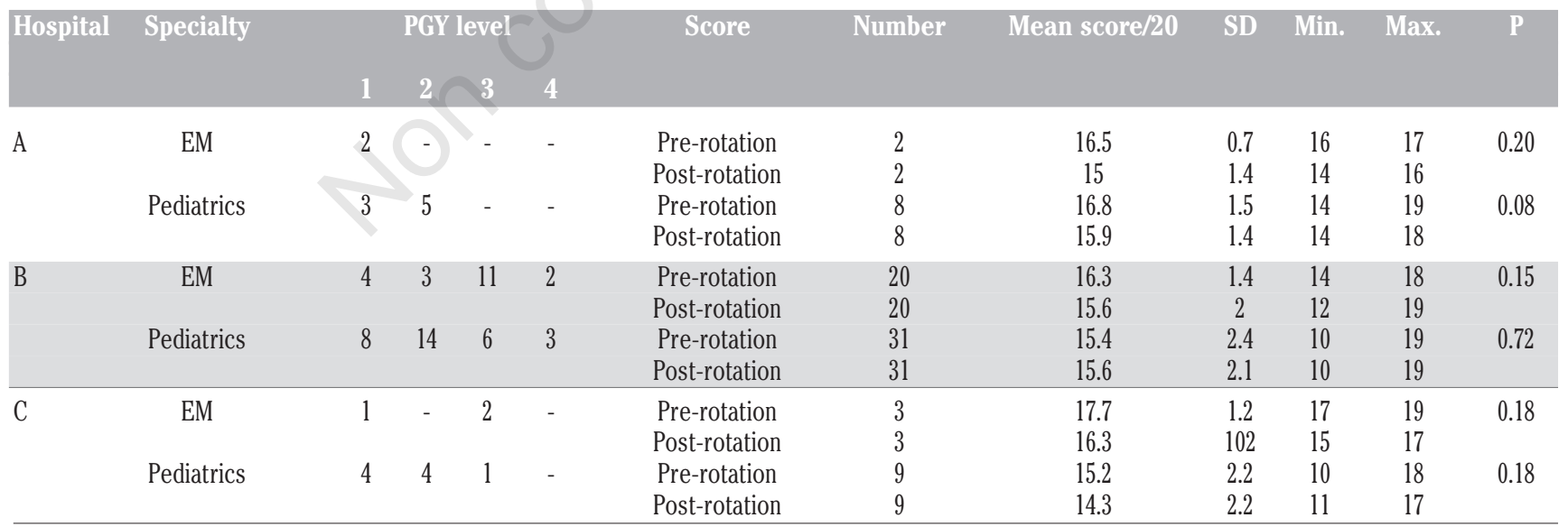

PGY, post-graduate year; SD, standard deviation; EM, emergency medicine.

Table 3. Residents' pre- and post-rotation scores according to the specialty.

\begin{tabular}{lcccccc} 
Specialty & Score & Number & Mean/20 & SD & Min. & \\
\multirow{2}{*}{ EM } & Pre-rotation & 25 & 16.5 & 1.4 & 14 & 19 \\
& Post-rotation & 25 & 15.6 & 1.8 & 12 & 19 \\
\multirow{2}{*}{ Pediatrics } & Pre-rotation & 48 & 15.6 & 2.2 & 10 & 19 \\
& Post-rotation & 48 & 15.4 & 2 & 10 & 19 \\
\hline
\end{tabular}

SD, standard deviation; EM, emergency medicine. 
Table 4. Residents pre- and post-rotation scores according to centers.

\begin{tabular}{|c|c|c|c|c|c|c|c|c|c|}
\hline Hospital & Annual PEM visits (n) & PEM consultants (n) & Score & Number & Mean/20 & SD & Min. & Max. & $\mathbf{P}$ \\
\hline A & 15,000 & 12 & $\begin{array}{l}\text { Pre-rotation } \\
\text { Post-rotation }\end{array}$ & $\begin{array}{l}10 \\
10\end{array}$ & $\begin{array}{l}16.7 \\
15.7\end{array}$ & $\begin{array}{l}1.3 \\
1.3\end{array}$ & $\begin{array}{l}14 \\
14\end{array}$ & $\begin{array}{l}19 \\
18\end{array}$ & 0.02 \\
\hline B & 100,000 & 20 & $\begin{array}{l}\text { Pre-rotation } \\
\text { Post-rotation }\end{array}$ & $\begin{array}{l}51 \\
51\end{array}$ & $\begin{array}{l}15.8 \\
15.6\end{array}$ & $\begin{array}{c}2.1 \\
2\end{array}$ & $\begin{array}{l}10 \\
10\end{array}$ & $\begin{array}{l}19 \\
19 \\
\end{array}$ & 0.59 \\
\hline $\mathrm{C}$ & 65,000 & 8 & $\begin{array}{l}\text { Pre-rotation } \\
\text { Post-rotation }\end{array}$ & $\begin{array}{l}12 \\
12\end{array}$ & $\begin{array}{l}15.8 \\
14.8\end{array}$ & $\begin{array}{l}2.2 \\
2.2\end{array}$ & $\begin{array}{l}10 \\
11\end{array}$ & $\begin{array}{l}19 \\
17\end{array}$ & 0.04 \\
\hline
\end{tabular}

PEM, pediatric emergency medicine; SD, standard deviation.

what similar in all three hospitals. A tertiary hospital is a center which caters to patients with complex disorders such as hematology, oncology, metabolic, immunodeficiency syndrome, neurologic disorders, congenital heart diseases, trauma and many general pediatric diseases. Even though our study may suggest residents' scientific knowledge did not improve after one month of rotation in PEM and attendance at four didactic academic sessions, we certainly cannot ignore the need for further evaluation of our training programs. Training requirements have to be periodically revised as educational weaknesses are identified, mainly by cross-sectional surveys of program directors. These surveys may be limited by recall bias and do not focus on measures of quality, such as quantifying clinical and procedural skills. ${ }^{17-19}$ Quantitative information such as number of patients seen, resuscitations and procedures performed enables program directors to identify training/trainee deficits, provide real-time feedback to the residents, and make real time changes. If important skills and knowledge cannot be attained during the rotation, then simulated resuscitations and standardized patient encounters may be used to supplement this experience. Monitoring the types of resident clinical encounters allows the program director to further tailor the didactic and interactive components of the curriculum to fill in the gaps. For example, simulation is useful for teaching high-acuity, low-frequency situations such as pediatric resuscitation. While many centers may already be using simulation to teach resuscitation and other highacuity events, this study suggests an important need for interactive educational experiences to teach additional skills or concepts that have low or no frequency during training..$^{20-22}$

The efficacy of an online didactic curriculum in improving knowledge acquisition among non EM, EM rotating residents and medical students during their EM rotations was established. After exposure to an online didactic curriculum, rotating residents demonstrated a significant increase in EM knowledge and reported a high level of satisfaction with the didactic program. ${ }^{23,24} \mathrm{~A}$ recent survey data show that $58 \%$ of rotating residents in academic EDs currently attend EM resident confer- ences, ${ }^{25}$ and only $7 \%$ would prefer attending the standard EM residency core conferences. ${ }^{26}$ It is difficult to predict didactic topics desired by rotating residents based solely on their respective medical specialties. ${ }^{27} \mathrm{~A}$ learner centered approach, ${ }^{28}$ allowing the resident to pick from a selection of didactic subjects, may be an appropriate solution. The community hospital provides the residents with exposure to the private practice environment, and its large children's Emergency Department (ED) provides pediatric EM experience. For those programs that use multiple training hospitals, identifying the types of patient encounters in each hospital may also help direct the residency curriculum.

According to international data, $30-40 \%$ of ED patients present with semi urgent or nonurgent conditions, ${ }^{29}$ the care of less acute complaints is a cornerstone of PEM practice. ${ }^{30}$ Assuming that our training model is similar to others, an alarm should be raised because the care of lower-acuity conditions may be a training deficit. As the use of urgent care centers and triage physicians in EDs increases; resident exposure to lower-acuity patients likely will decreases. Residents must also be afforded the opportunity to supervise and collaborate with midlevel providers. ${ }^{31-33}$ Ensuring adequate exposure to low-acuity conditions should be a priority for Program Directors and should affect the staffing plans for EDs with PEM residency programs. There is a strong need for continued research in the educational needs of residents and evaluation of educational experiences in PEM training programs. Further studies, possibly incorporating simulation or actual patient encounters, should be performed to determine whether this improved knowledge results in better patient care outcomes.

\section{Limitations}

We aimed to evaluate the improvement in knowledge acquisition as measured by scores on a multiple-choice test. We acknowledge that the goal of any curriculum is to achieve true competency among learners, only a facet of which is test-taking ability and medical knowledge. Furthermore, our results might differ if residents had been assessed by other means like objectively structured clinical examina- tion. Finally, these results represent the experience of only these institutions. The conclusions may not be generalizable to other centers. The pretest was performed at the start and the same questions repeated at the end of the rotation. Some residents could have studied these questions checking for the correct answer, though none of them score the full marks on the post test. Thus, the majority of interactions between the educational supervisors in the ED and the study participants likely occurred without confounding knowledge transmission. While there was no way to standardize clinical teaching, shift schedules for all participants were made based on routine scheduling requirements by a consultant who was not involved in the study. Shift schedules for PEM faculty were made by a faculty member who had no knowledge of the study or study participant shift schedule. Therefore, there is no reason to suspect that residents had any significant differences in clinical teaching or patient care experiences. We therefore feel that the educational experience of residents was an accurate representation of learning by clinical practice and teaching alone. Future studies should include a larger sample size and multiple institutions.

\section{Conclusions}

After one month of rotation in PEM, we observed a decrease in the post-rotation test scores as compared to the pre-rotation scores. Since the reasons for the lower scores could not be identified by this study, educational deficiencies should be identified and perhaps a focused teaching and allotted study time to optimize the residents educational objective could be advised.

\section{References}

1. Sanders AB, Kobernick ME. Educating internists in emergency medicine. West $\mathrm{J}$ Med 1984;141:534-7. 
2. Branzetti JB, Aldeen AZ, Courtney DM. Educational orphans: a survey of emergency medicine residency directors on didactics for rotating residents in the emergency department. Acad Emerg Med 2009;16:48.

3. Kessler CS, Marcolini EG, Schmitz G, et al. Off-service resident education in the emergency department: outline of a national standardized curriculum. Acad Emerg Med 2009;16:1325-30.

4. Rodenberg H. Education in accident and emergency medicine for senior house officers: review and recommendations. J Accid Emerg Med 1996;13:238-42.

5. Pena ME, Snyder BL. Pediatric emergency medicine. The history of a growing discipline. Emerg Med Clin N Am 1995;13:23553.

6. Barkin RM. Pediatric emergency medicine comes of age. Acad Emerg Med 1994;1:12930 .

7. Abramo TJ. Pediatric emergency medicine fellowship programs. Pediatr Emerg Care 1997;13:169-77.

8. Izsak E. Pediatric emergency medicine fellowship programs. Pediatr Emerg Care 1994;10:121-6.

9. Jaffe DM. Research in emergency medical services for children. Pediatrics 1995; 96:191-4.

10. Cook RT Jr. The Institute of Medicine report on emergency medical services for children: thoughts for emergency medical technicians, paramedics, and emergency physicians. Pediatrics 1995;96:199-206.

11. Asch SM, Weigand JV. A pediatric curriculum for emergency medicine training programs. Ann Emerg Med 1986;15:19-27.

12. Singer JI, Hamilton GC. Objectives to direct the training of emergency medicine residents in pediatric emergency medicine. J Emerg Med 1993;11:211-8.

13. Boyle MF, Eilers MA, Hunt RL, et al. Objectives to direct the training of emergency medicine residents on off-service rotations: emergency medical services. J Emerg Med 1990;8:791-5.

14. Asch SM, Weigand JV. A pediatric curricu- lum for emergency medicine training programs. Ann Emerg Med 1986;15:19-27.

15. Saudi Commission for Health Specialties, fellowships. Available from: http://www.scfhs.org.sa/en/education/Train ingAndRecognition/HighEduProgs/Fellows hipPrograms/Pages/default.aspx

16. Saudi Specialty Certificate of Pediatrics and Emergency Medicine training programs. Available from: http://www.kfshrc.edu.sa/ATA/TrainingPro grams.pdf

17. Accreditation Council for Graduate Medical Education. 2007 Residency Review Committee Program, requirements for Emergency Medicine. Available from: http://www.acgme.org/acgmeweb/ tabid/131/ProgramandInstitutionalAccredi tation/Hospital-BasedSpecialties/ EmergencyMedicine.aspx

18. Accreditation Council for Graduate Medical Education. 2007 Residency Review Committee Program, requirements for Pediatrics. Available from: http://www.acgme.org/acgmeweb/tabid/143 /ProgramandInstitutionalAccreditation/Me dicalSpecialties/Pediatrics.aspx

19. Chen S, Shofer F, Baren J. Emergency medicine resident rotation in pediatric emergency medicine: what kind of experience are we providing? Acad Emerg Med 2004;11:771-3.

20. Ludwig S, Fleisher G, Henretig F, Ruddy R. Pediatric training in emergency medicine residency programs. Ann Emerg Med 1982;11:170-3.

21. Ros SP, Cetta F, Ludwig S. Pediatric education in emergency medicine residency programs-10 years later. Pediatr Emerg Care 1993;9:143-5.

22. Biese KJ, Moro-Sutherland D, Furberg RD, et al. Using screen-based simulation to improve performance during pediatric resuscitation. Acad Emerg Med 2009;16 (Suppl 2):S71-5.

23. American Board of Emergency Medicine. Qualifying examination description and content specifications. Available from: https://www.abem.org/public/emergency- medicine-\%28em\%29-initial-certification/qualifying-examination/qualifyingexamination-description-and-contentspecificiations

24. Tintinalli J, Shofer F, Biese K, Phipps J. Toward a new paradigm: goal-based residency training. Acad Emerg Med 2011; 18:71-8.

25. Burnette K, Ramundo M, Stevenson M, Beeson MS. Evaluation of a web-based asynchronous pediatric emergency medicine learning tool for residents and medical students. Acad Emerg Med 2009;16 (Suppl 2):S46-50.

26. Branzetti JB, Aldeen AZ, Foster AW, Courtney DM. A novel online didactic curriculum helps improve knowledge acquisition among non-emergency medicine rotating residents. Acad Emerg Med 2011; 18:53-9.

27. Tenn-Lyn NA, LeBlanc VR, Bandiera GW. Can we predict what objectives off-service residents have for their emergency medicine rotations. Ann Emerg Med 2008;51: 516 (abstract).

28. Carter AJ, McCauley WA. Off-service residents in the emergency department: the need for learner centredness. CJEM 2003;5:400-5.

29. Niska R, Bhuiya F, Xu J. National hospital ambulatory medical care survey: 2007 emergency department summary. Natl Health Stat Rep 2010;26:1-32.

30. Perina DG, Beeson MS, Char DM, et al. The 2007 Model of the clinical practice of emergency medicine: the 2009 update. Acad Emerg Med 2011;18:e8-26.

31. Crane M, Guglielmo W. NPs and PAs. What's the malpractice risk? Med Econ 2000;77:205-8.

32. Henry G. Mid-levels: the staffing solution of the future? Available from: http://www.epmonthly.com/departments/co lumns/oh-henry/mid-levels-the-staffingsolution-of-the-future/

33. Henry G. Mid-level question calls for highlevel discourse. Emerg Physicians Mon 2011;18:39. 